\title{
O TECNOSTRESS EM PROFISSIONAIS DE SAÚDE NOS CUIDADOS DE SAÚDE PRIMÁRIOS
}

\author{
Cláudia Chaves \\ Doutoranda em Ciências de Enfermagem \\ Instituto de Ciências Biomédicas Abel Salazar \\ SIGMA - Phi Xi Chapter / CI\&DETS / \\ Politécnico de Viseu - Portugal \\ claudiachaves21@gmail.com \\ João Duarte \\ UICISA:E / CI\&DETS \\ Politécnico de Viseu - Portugal \\ Paula Nelas \\ UICISA:E / CI\&DETS \\ Politécnico de Viseu - Portugal \\ Emília Coutinho \\ SIGMA - Phi Xi Chapter/ UICISA:E / CI\&DETS / \\ Politécnico de Viseu - Portugal \\ Carla Cruz \\ UICISA:E / CI\&DETS \\ Politécnico de Viseu - Portugal \\ Rui Dionísio \\ SIGMA - Phi Xi Chapter / ESSV / Politécnico de Viseu \\ Unidade de Saúde Pública \\ ACES Dão Lafões - Portugal
}

Fecha de Recepción: 18 Agosto 2019

Fecha de Admisión: 25 Septiembre 2019

\section{RESUMO}

Enquadramento: 0 uso de tecnologias de comunicação e informação, com 0 recurso constante a aplicativos informáticos, no trabalho diariamente pode induzir a um estado psicológico negativo, também designado por tecnostress. Objetivos: Caracterizar o nível de tecnostress nos profissionais de saúde dos cuidados de saúde primários; identificar de que modo as variáveis sociodemográficas e 0 tecnostress se relacionam nos profissionais de saúde. Metodologia: Estudo quantitativo, transversal, descritivo e correlacional. 0 instrumento de recolha de dados utilizado foi questionário sociodemográfico e a Escala de Tecnostress (Carlotto \& Câmara, 2010). Resultados: A amostra é constituída por 100 profissionais de saúde, maioritariamente do sexo feminino (72,0\%), com uma idade média de 46,14 anos ( $\pm 10,72$ anos), $49,1 \%$ são enfermeiros e $28,1 \%$ médicos, com 


\section{O TECNOSTRESS EM PROFISSIONAIS DE SAÚDE NOS CUIDADOS DE SAÚDE PRIMÁRIOS}

menos tempo de exercício profissional na Unidade $(35,0 \%)$ e com anos de experiência profissional entre 16-22 (34,0\%). São os profissionais de saúde do sexo masculino os que revelam mais tecnostress, à exceção do ceticismo $(0 \mathrm{M}=52,53)$, onde foram as mulheres que pontuaram mais. Os homens revelam mais uso compulsivo das Tecnologias de Informação e Comunicação. Conclusão: Os dados obtidos sugerem a implementação e monitorização de medidas que potenciem uma melhor utilização das tecnologias informáticas de comunicação no local de trabalho em profissionais de saúde nos cuidados de saúde primários.

Palavras-chave: profissionais de saúde; cuidados de saúde primários; tecnostress.

\section{ABSTRACT}

\section{Tecnostress in health professionals in primary health care.}

Background: The use of communication and information technologies, with the constant use of computer applications, in daily work can induce a negative psychological state, also called tecnostress. Objectives: To characterize the level of technostress in the health professionals of primary health care; identify how the socio-demographic variables and the technostress are related in health professionals. Methodology: Quantitative, transversal, descriptive and correlational study. The data collection instrument used was a sociodemographic questionnaire and the Tecnostress Scale (Carlotto \& Câmara, 2010). Results: The sample consisted of 100 health professionals, mostly female $(72.0 \%)$, with a mean age of 46.14 years ( \pm 10.72 years), $49.1 \%$ were nurses and $28.1 \%$ doctors, and $35.0 \%$ with less years in the health unit, professional experience between 16-22 years $(34.0 \%)$. It is male health professionals who show more technostress, with the exception of skepticism (MR = 52.53), where the women scored more. Men show more compulsive use of Information and Communication Technologies. Conclusion: We consider important the implementation and monitoring of measures that promote better use of information technology in the workplace in health professionals in primary health care.

Keywords: health professionals; primary health care; tecnostress.

\section{INTRODUÇÃO}

Nas últimas décadas, as mudanças tecnológicas têm orientado as organizações para a era da informação, de tal forma que se propõe uma adequação às rápidas mudanças e a encarar positivamente as renovações tecnológicas (Carlotto \& Câmara, 2010; Silva, Queirós, Cameira, 2016). No contexto laboral contemporâneo, os trabalhadores utilizam cada vez mais as tecnologias de comunicação e informação para poderem acompanhar e interagir com os vários estádios de conceção, elaboração de produtos e serviços. Assim, novas características são exigidas, originado um novo conceito e perfil de trabalhador, o qual deve saber lidar com concepções, processamento e interpretação dados informaticamente. 0 novo trabalhador tem que se adaptar às novas linguagens, que se modificam constantemente, sendo a área tecnológica responsável por $80 \%$ dos postos de trabaIho (Souto, 2003).

A consequência direta do avanço tecnológico é que os trabalhadores passam a depender das tecnologias para executarem as suas atividades laborais, sendo-Ihes exigido mais capacitação para se adequarem aos novos meios, bem como mais celeridade e mais dinamismo, em qualquer condição, como premissa organizacional (Carlotto \& Câmara, 2010). Segundo os mesmos autores, é neste contexto de várias exigências organizacionais de atualização e capacitação que 0 trabalhador tem de saber lidar com novas informações, vendo-se cada vez mais perante inúmeras situações às quais necessita de se adaptar. Deste modo, o trabalho requer maior exigência cognitiva e, por consequência, maior é a sobrecarga nos seus processos mentais. Uma sobrecarga resultante de estímulos internos (necessidades e perspetivas pessoais) e externos (exigências dos sistemas produti- 
vos e pressão social). Face a estas características da atual lógica de trabalho, o stresse é um fenómeno cada vez mais comum entre os trabalhadores (Ferreira, 2014). De acordo com o mesmo autor, com a inclusão da tecnologia no ambiente de trabalho, mais profissionais estão propensos ao stresse tecnológico, designado de tecnostresse, cujo conceito está diretamente relacionado aos efeitos psicossociais negativos do uso das Tecnologias da informaç̧ão e comunicação (TIC). Nível do tecnostress perante 0 trabalho diário com aplicativos informáticos pode induzir é um estado psicológico negativo que se relaciona com o uso de tecnologias de comunicação e informaç̧ão ou de ameaça do seu uso futuro. Trata-se de um fenómeno psicossocial constituído por quatro dimensões: descrença, ansiedade, fadiga e ineficácia (Carlotto \& Câmara, 2010). É um problema que possui uma componente psicológica de base, mas que acaba por afetar o corpo de uma forma geral, principalmente 0 estado anímico, uma vez que pode resultar em níveis elevados de stresse, ansiedade e num estado anímico baixo.

As inovações e as mudanças tecnológicas têm uma dupla face, ou seja, se, por um lado, trazem benefícios económicos para as organizações e para muitos aspectos relacionados com o conteúdo do posto de trabalho; por outro lado, parecem resultar em problemas humanos e sociais com consequências ao nível da saúde mental do trabalhador (Carlotto, 2011). A mesma autora refere que a tecnologia, contudo, é neutra, ou seja, por si mesma, não origina efeitos negativos nem positivos. A qualidade e a intensidade dos seus efeitos dependem de vários fatores, designadamente as exigências e a falta de recursos gerados no trabalho, assim como as crenças nas próprias capacidades e competências para enfrentar com êxito as mudanças tecnológicas. Neste cenário, o stresse é um dos fenómenos cada vez mais comum entre os trabalhadores, refletido nos casos de transtornos psicossomáticos e doenças ocupacionais evidentes e/ou emergentes. Carlotto (2011) realizou um estudo com 668 trabalhadores, tendo aplicado a escala de Tecnostress (RED/TIC), uma escala acerca das Estratégias de Enfrentamento (COPE) além de um questionário contendo variáveis sociodemográficas e laborais. Os resultados demonstraram que as mulheres apresentam médias mais elevadas nas dimensões de descrença, ansiedade e ineficácia. Em relação às variáveis sociodemográficas, foram identificadas diferenças entre homens e mulheres nas variáveis idade e escolaridade; e, quanto às laborais, na utilização de e-mail e chat. As estratégias para enfrentarem o tecnostress utilizadas por homens e mulheres também revelaram diferenças significativas, ou seja, os homens apresentaram médias mais elevadas em termos de enfrentamento ativo, planeamento, supressão de atividades concomitantes, enquanto as mulheres revelaram médias mais elevadas nas estratégias de suporte social e emocional, religiosidade, foco na emoção, negação, desligamento comportamental e desligamento mental.

Partindo deste enquadramento, equacionam-se as seguintes questões de investigação: (i) Qual o nível de tecnostress nos profissionais de saúde de cuidados de saúde primários? (ii) De que modo as variáveis sociodemográficas se relacionam com tecnostress dos profissionais de saúde de Cuidados de Saúde Primários? 0 objetivo principal deste estudo consiste em avaliar o nível de tecnostress dos profissionais de saúde de Cuidados de Saúde Primários e como objetivo específico, identificar de que modo as variáveis sociodemográficas se relacionam com tecnostress dos profissionais de saúde dos Cuidados de Saúde Primários.

\section{MATERIAL E MÉTODOS}

Estudo quantitativo, transversal, descritivo e correlacional. Para a realização deste estudo, considerou-se como população os profissionais a exercerem funções em cuidados de saúde primários, nos centros de saúde de um agrupamento de centros de saúde no interior centro de Portugal (com um total de 235 questionários distribuídos a todos os profissionais), extraiu-se a amostra não pro- 


\section{O TECNOSTRESS EM PROFISSIONAIS DE SAÚdE NOS CUIDADOS DE SAÚdE PRIMÁRIOS}

babilística por conveniência, uma vez constituída por 100 profissionais de saúde de Cuidados de Saúde Primários que devolveram os questionários totalmente preenchidos. 0 instrumento de recoIha de dados utilizado foi questionário sociodemográfico e a Escala de Tecnostress (Carlotto \& Câmara, 2010). A Escala de Tecnostress (Carlotto \& Câmara, 2010) avalia o tecnostress a partir de um modelo teórico de quatro dimensões: fadiga, ansiedade, descrença e ineficácia. Trata-se de uma escala desenvolvida pela equipa de investigadores da WONT - Work, Organization \& New Technologies del Departamento de Prevención Psicosocial de la Universitat Jaume I (CastellónEspanha), e tem como base o modelo teórico da Psicologia Social do Trabalho, no campo da intervenção psicossocial e saúde do trabalhador. Baseia-se no modelo RED (Recursos, Emoções/Experiência, Demandas), que perceciona stresse como um processo interacional entre as exigências do ambiente de trabalho, os recursos ambientais e pessoais de que dispõe cada profissional para poder enfrentá-Io (Carlotto \& Câmara, 2010). A Escala de Tecnoestress para os Usuários de Tecnologias de Informação e Comunicação consiste de 16 questões, subdivididas em quatro dimensões: 1) ceticismo (itens 1, 2, 3, 4), 2); fadiga (itens 5, 6, 7, 8); 3) ansiedade (itens 9, 10, 11 , 12); e 4) ineficácia (itens $13,14,15,16$ ) e acrescenta ainda 6 itens de classificação de uso compulsivo. Todos os itens são avaliados numa escala tipo Likert de 6 pontos, variando de zero (nunca) a seis (sempre). A escala original apresentou alfas de 0,93 para descrença, 0,92 para fadiga, 0,83 para ansiedade e 0,84 para ineficácia. 0 score final de cada um dos atributos é dado pela média das respostas de seus itens, sendo que médias elevadas nas dimensões são indicadores de tecnoestress. A adaptação da escala (tradução para o Português do Brasil) e o estudo prévio das propriedades psicométricas (validade de conteúdo, validade de constructo e confiabilidade), foram realizados por Carlotto e Câmara (2010), tendo todos os factores apresentado um alfa de Cronbach superior a 0,70 (Descrença $\alpha=0,74$; Fadiga $\alpha=0,89$; Ansiedade $\alpha=0,77$ Ineficácia $\alpha=0,80$ ). Assim, os resultados mostram que a escala apresenta validade fatorial e consistência interna adequadas para avaliar o tecnoestress em profissionais. Foi acrescentado um item (14) "é difícil trabalhar com as tecnologias de informação e comunicação".

Nos procedimentos éticos, no desenvolvimento da investigação foram salvaguardados os direitos dos participantes e os princípios éticos fundamentais tal foi conduzido, através do consentimento informado, que contemplam a informação referente ao estudo, como, o tema e o objetivo da investigação, pedido de colaboração para o preenchimento e referência à necessidade de resposta a todas as questões, para que este não fosse eliminado, garantia de anonimato e confidencialidade das respostas, disponibilização dos resultados, agradecimento da colaboração e disponibilidade dispensadas.

Para a análise dos dados, todo o tratamento estatístico foi processado através do programa SPSS (Statistical Package for the Social Sciences) versão 24.0 para Windows. E recorreu-se à determinação das frequências absolutas e percentuais, médias, medidas dispersão, como a amplitude de variação, o coeficiente de variação e 0 desvio padrão, bem como a assimetria, achatamento e 0 coeficiente de correlação de Pearson. Em termos de análise inferencial, recorreu-se ao teste de U Mann Whitney, análise de variância a um fator ou Teste de Kruskall Wallis e teste de qui quadrado.

\section{RESULTADOS}

Na globalidade da amostra, maioritariamente são do sexo feminino $(72,0 \%)$, apresentam uma idade mínima de 22 anos e uma máxima de 66 anos, ao que corresponde uma idade média de 46,14 anos ( $\pm 10,72$ anos). Os homens, em média ( $M=48,04$ anos12.77 anos), são ligeiramente mais velhos do que as mulheres de 45,50 anos ( \pm 9.81 anos). Verifica-se que relativamente à experiência profissional, $34,0 \%$ do total da amostra possui entre 16-22 anos de experiência profissional, $33,0 \%$ de participantes possuem $\leq 15$ anos de exercício profissional, e dos $33,0 \%$ dos profissionais 
de saúde que têm $\geq 23$ anos de exercício profissional. Constata-se que em relação à profissão, a maioria dos participantes são enfermeiros (49,1\%), seguindo-se os médicos $(28,1 \%)$, e $14,0 \%$ são assistentes operacionais.

Verifica-se que prevalecem os participantes com menos tempo de exercício profissional na Unidade atual $(35,0 \%)$, dos quais $42,9 \%$ são homens e $31,9 \%$ mulheres, seguindo-se os que exercem na Unidade atual há mais anos $(34,0 \%)$, onde a percentagem mais elevada é a das mulheres $(34,7 \%)$. É também de referir que $31,0 \%$ dos participantes exercem na Unidade atual entre os 4-9 anos, dos quais $25,0 \%$ são homens e $33,3 \%$ mulheres. Ainda em relação ao tempo de exercício profissional, os profissionais de saúde apresentam um tempo de serviço mínimo de um ano e um máximo de 48 anos, ao que corresponde uma média de 19,36 anos ( $\pm 10,94$ anos). Para 0 sexo masculino, o tempo de exercício profissional mínimo é de 2 anos e o máximo de 42 anos, enquanto para o feminino 0 tempo de serviço mínimo é de 1 ano, sendo 0 máximo de 48 anos. Os homens, em média, têm mais tempo de serviço ( $M=22,07$ anos de tempo de serviço $\pm 12,39$ anos) que as mulheres ( $M=18,31$ anos de exercício profissional $\pm 10,22$ anos). Os coeficientes de variação indiciam uma dispersão alta face às médias encontradas.

Os resultados relativos ao tecnostress indicam que 0 valor médio mais elevado corresponde ao uso compulsivo ( $M=2,401,00)$, onde 0 mínimo encontrado é 0,00 e 0 máximo 4,67, seguindo-se a fadiga ( $M=1,851,15)$, sendo 0 valor mais baixo 0 obtido na ineficácia $(M=1,320,96)$. Os valores de assimetria e curtose apresentam curvas não enviesadas, mas platicurticas para 0 uso compulsivo. Quanto aos coeficientes de variação, registam-se dispersões elevadas em torno do valor médio.

Observa-se que a maioria dos profissionais de saúde $(28,0 \%)$ refere que, com 0 passar do tempo, as tecnologias os interessam cada vez menos. Relativamente ao facto de cada vez se sentirem menos motivados para 0 uso das tecnologias, $31,0 \%$ diz que nunca isso acontece. Verifica-se que $28,0 \%$ dos profissionais de saúde admitem que se tornaram algumas vezes mais céticos em relação à contribuição das tecnologias para 0 seu trabalho; $34,0 \%$ referem que quase nunca duvidam do significado do trabalho com as tecnologias; $32,0 \%$ consideram que algumas vezes é difícil descontrair após um dia de trabalho a usar tecnologias, bem como, em idêntica percentagem, sentem-se algumas vezes esgotados quando acabam de trabalhar com tecnologias. Para 30,0\% dos profissionais de saúde, raramente se sentem tão cansados quando acabam de trabalhar com tecnologias que não Ihes permite fazer mais nada. Por sua vez, 37,0\% dos participantes admitem que raramente é difícil concentrarem-se depois de trabalharem com tecnologias; $26,0 \%$ afirmam que nunca se sentem tensos e/ou ansiosos quando trabalham com tecnologias, contrariamente a 23,0\% que admitem sentir-se tensos e/ou ansiosos algumas vezes.

Segundo $34,0 \%$ dos participantes, assusta-0s algumas vezes pensar que podem perder grande quantidade de informação com o uso inadequado das tecnologias; $27,0 \%$ quase nunca duvidam das tecnologias com medo de cometer erros, enquanto $20,0 \%$ admitem que isso ocorre algumas vezes. Em 29,0\% dos participantes nunca se sentem incomodados, irritados e impacientes por trabalharem com tecnologias, contudo 17,0\% afirmam que isso ocorre algumas vezes. Na opinião de $31,0 \%$ dos participantes nunca se sentem ineficazes quando utilizam tecnologias, contrariamente a $15,0 \%$ que consideram que essa situação acontece algumas vezes. Constata-se, que para $27,0 \%$ quase nunca é difícil trabalharem com as tecnologias de informação e comunicação, enquanto $20,0 \%$ admitem que essa dificuldade ocorre algumas vezes.

Para $41,0 \%$ dos profissionais de saúde, as pessoas nunca dizem que são utilizadores ineficazes; 33,0\% também afirmam que nunca sentem insegurança ao realizarem as suas tarefas quando usam as tecnologias, enquanto $13,0 \%$ admitem que Ihes acontece tal situação algumas vezes; $31,0 \%$ dos profissionais de saúde pensam algumas vezes que utilizam em excesso as tecnologias 


\section{O TECNOSTRESS EM PROFISSIONAIS DE SAÚdE NOS CUIDADOS DE SAÚdE PRIMÁRIOS}

na sua vida, enquanto $26,0 \%$ afirmam que esse pensamento Ihes ocorre raramente. A maioria dos participantes $(34,0 \%)$ refere que utilizam, algumas vezes, continuamente as tecnologias mesmo fora do seu horário de trabalho, enquanto $21,0 \%$ diz fazê-lo frequentemente. Segundo a maioria dos participantes $(33,0 \%)$, pensam nas tecnologias frequentemente (por exemplo telemóvel, correio eletrónico, etc.) mesmo fora do horário de trabalho algumas vezes, o que para 25,0\% acontece frequentemente. No que diz respeito a sentirem-se ansiosos quando não têm acesso às tecnologias (telemóvel, correio eletrónico, etc.), verifica-se que a percentagem mais eleva corresponde aos participantes que mencionam que se sentem dessa maneira algumas vezes, já para 19,0\% é frequente isso ocorrer. Constata-se também que em $22,0 \%$ dos profissionais de saúde um impulso interno obriga-os raramente a utilizá-las em qualquer lugar e em qualquer momento, já para 23,0\% essa ocorrência surge algumas vezes. Na perspetiva de $28,0 \%$ dos participantes, nunca dedicam mais tempo às tecnologias do que em estar com os amigos/ família ou a atividades de lazer, enquanto $17,0 \%$ admite que dedicam algumas vezes esse tempo às tecnologias em detrimento do convívio social.

Pelos resultados obtidos no teste binominal, pode afirmar-se que na globalidade os valores se situam na opção de resposta nunca, sugerindo que os profissionais de saúde não sentem tecnostress.

Na relação entre o tecnostress e as variáveis sociodemográficas dos profissionais de saúde de Cuidados de Saúde Primários, constata-se que, na globalidade, são os profissionais de saúde do sexo masculino os que revelam mais tecnostress, à exceção do ceticismo $(0 M=52,53)$, onde foram as mulheres que pontuaram mais. Os homens revelam mais uso compulsivo das Tecnologias de Informação e Comunicação. Todavia, constata-se que não existem diferenças estatisticamente significativas.

De acordo com os resultados reportados na Tabela 1, pode afirmar-se que, na globalidade, são os profissionais de saúde com mais tempo de exercício profissional na Unidade atual os que manifestam mais tecnostress, com um valor médio mais elevado no ceticismo. Constata-se que, os profissionais com menos tempo a exercerem na Unidade são os que revelam índices mais baixos de tecnostress, registando-se diferenças estatisticamente significativas no ceticismo $(p=0,007)$ e na ineficácia $(p=0,032)$.

Tabela 1: Tempo de serviço na Unidade actual e o Tecnostress

\begin{tabular}{|c|c|c|c|c|c|c|c|c|}
\hline $\begin{array}{l}\text { Tempo de } \\
\text { serviço na } \\
\text { Unidade }\end{array}$ & \multicolumn{2}{|c|}{$\leq 3$ anos } & \multicolumn{2}{|c|}{ 4-9 anos } & \multicolumn{2}{|c|}{$\geq 10$ anos } & \multirow{2}{*}{ f } & p \\
\hline & Gódi & & Gódi & & Gódi & $\mathrm{Dm}$ & & \\
\hline Ceticismo & 1,43 & 1,22 & 1,56 & 1,19 & 2,29 & 1,12 & 5,21 & 0,007 \\
\hline Fadiga & 1,83 & 1,26 & 1,72 & 1,19 & 1,98 & 1,00 & 0,41 & 0,665 \\
\hline Ansiedade & 1,54 & 1,19 & 1,72 & 1,12 & 1,88 & 1,15 & 0,77 & 0,464 \\
\hline Ineficácia & 1,02 & 0,88 & 1,30 & 0,93 & 1,63 & 1,00 & 3,55 & $\mathbf{0 , 0 3 2}$ \\
\hline $\begin{array}{l}\text { Uso } \\
\text { compulsivo }\end{array}$ & 2,44 & 1,00 & 2,48 & 0,98 & 2,28 & 1,03 & 0,34 & 0,710 \\
\hline
\end{tabular}

\section{DISCUSSÃO}

Os procedimentos metodológicos tiveram como linha orientadora os objetivos traçados, contudo há algumas limitações a referir, como o facto de se tratar de um estudo transversal, logo com a impossibilidade de estabelecerem relações causais e também recolha de informação ter por base 
um processo de amostragem não garantiu a representatividade da população em estudo, o que não possibilita que se extrapolem os resultados obtidos. Contudo, lançam-se pistas para a realização de estudos futuros com amostras mais dilatadas e numa área geográfica mais abrangente.

Pelos resultados pode afirmar-se que na globalidade os valores se situam na opção de resposta nunca, sugerindo que os profissionais de saúde não sentem tecnostress, resultados também apurados por Silva, Queirós, Cameira (2016), em população idêntica. São os profissionais de saúde com mais tempo de exercício profissional na Unidade atual os que manifestam mais tecnostress, com um valor médio mais elevado no ceticismo. Constata-se que, os profissionais com menos tempo a exercerem na Unidade actual são os que revelam índices mais baixos de tecnostress, registando-se diferenças estatisticamente significativas no ceticismo $(p=0,007)$ e na ineficácia $(p=0,032)$. Também se verificou que os profissionais de saúde do sexo masculino revelam mais tecnostress, à exceção do ceticismo $(0 M=52,53)$, onde foram as mulheres que pontuaram mais, o que não está em conformidade com o estudo de Carlotto (2011) cujos resultados demonstraram que as mulheres apresentam médias mais elevadas nas dimensões de descrença, ansiedade e ineficácia.

\section{CONCLUSÃO}

Deste modo, em termos de perfil sociodemográfico e profissional, concluiu-se que se trata de uma amostra maioritariamente feminina com uma idade média de 46,14 anos ( $\pm 10,72$ anos), estando em maioria os enfermeiros, secundados pelos médicos. A maioria dos inquiridos possui entre 16-22 anos de experiência profissional, prevalecendo os participantes com menos tempo de exercício profissional na atual Unidade.

Quanto aos resultados alcançados em relação ao tecnostress, concluiu-se que os profissionais de saúdes revelam principalmente uso compulsivo das tecnologias de informação e comunicação no local de trabalho e a fadiga, porém sentem menos ineficácia. Na globalidade, os profissionais de saúde não sentem tecnostress, sendo os profissionais de saúde com mais tempo de exercício profissional na Unidade os que manifestam mais tecnostress, sobretudo ceticismo; os profissionais com a exercerem há menos tempo na Unidade revelam índices mais baixos de tecnostress. Também se verificou que os profissionais de saúde do sexo masculino revelam mais tecnostress, à exceção do ceticismo, onde sobressaíram as mulheres.

No que se refere a investigações futuras, sugere-se estudar as interações e as relações entre 0 trabalho e os vínculos afetivos e familiares e suas repercussões na satisfação dos profissionais de saúde com 0 trabalho. É fundamental consciencializar os profissionais de saúde para a reflexão sobre a realidade vivenciada que, dada a sua dinâmica, se encontra em permanente transformação, impondo um ajustamento constante para que a qualidade da prestação de cuidados seja de qualidade.

\section{REFERÊNCIAS BIBLIOGRÁFICAS}

Carlotto, M. S. (2011). Tecnoestresse: Diferenças entre homens e mulheres. Revista Psicologia: Organizações e Trabalho, 11(2), 51-64. Acedido em http://pepsic.bvsalud.org/scielo.php?script=sci_arttext\&pid=\$1984-66572011000200005

Carlotto, M. S., \& Câmara, S. G. (2010). Tradução, adaptação e exploração de propriedades psicométricas da escala de tecnoestresse (RED/TIC). Psicologia em Estudo, 15(1), 171-178. https://dx.doi.org/10.1590/S1413-73722010000100018

Ferreira, A. P. C. (2014). Tecnologia de informação, controle e mundo do trabalho: pensar tecnologia na ótica do trabalhador. Revista Eletrônica de Ciências Sociais, 11, 14-24. Acedido em http://www.periodicos.ufpb.br/ojs2/index.php/caos/article/view/46917 


\section{O TECNOSTRESS EM PROFISSIONAIS DE SAÚdE NOS CUIDADOS DE SAÚDE PRIMÁRIOS}

Silva, M., Queirós, C., \& Cameira, M. (2016). Saúde no trabalho: Tecnostress e burnout em enfermeiros. International Journal on Working Conditions, 12, 54-70. Acedido em http://ricot.com.pt/artigos/1/IJWC.12_Silva,Queiros\&Cameira_54.70.pdf

Souto, S. M. de 0. (2003). 0 profissional da informação frente às tecnologias do novo milênio e as exigências do mundo do trabalho. In IV CINFORM: Encontro Nacional de Ciência da Informação, IV Anais: Ciência da Informação: Fronteiras e tendências. Salvador: UNICAMP. Acedido em http://www.cinform.ufba.br/iv_anais/artigos/texto16.htm 